\title{
STRATEGIES FOR TURKISH AUTOMOTIVE BY-PRODUCTS INDUSTRY
}

\author{
HAKAN BÜTÜNER, SUZAN SARPER ÖZCAN \\ Industrial Management and Engineering Co, Istanbul, Turkey
}

\section{ABSTRACT}

Automotive industry is comprised of the industry which produces vehicles, and the industry which, as dominated by Small and Medium Scale Enterprises, produces parts, modules, and systems of such vehicles according to the technical documentation. Having been established through technical and economic supports of the major manufacturers, and developed in the course of time, the by products industry is involved its activities as a significant potential for the domestic economy at present. In this article, general characteristics of Turkish automative by products industry, its weak aspects, aspects need to be improved, and dominant strategies that need to be considered for the improvement are discussed.

\section{JEL CLASSIFICATION \& KEYWORDS}

- O52 | Industry | Analysis | Strategy | Turkish automotive - By-products

\section{INTRODUCTION}

Automotive industry is comprised of the industry which produces vehicles, and the industry which, as dominated by Small and Medium Scale Enterprises, produces parts, modules, and systems of such vehicles according to the technical documentation. Having been established through technical and economic supports of the major manufacturers, and developed in the course of time, the by products industry is involved its activities as a significant potential for the domestic economy at present.

In this article, general characteristics of Turkish automative by products industry, its weak aspects (such as, high production costs due to low capacity utilization, excessive number of companies and excess capacity, etc.), aspects need to be improved (such as, lack of strategies for sectoral developments and uncertain vision, lack of sufficient specialization, etc.), and dominant strategies (about corporate skills, organizational structures of companies, new financing models, new marketing strategies, etc.) that need to be considered for the improvement are discussed.

General characteristics of Turkish automotive by products industry

Turkish automotive by products industry has reached to a level where it can supply almost all of the following parts and components:

- Complete motors and motor parts,

- transmission bodies,

- braking systems and parts,

- hydraulic and pneumatic parts,

- suspension parts,

- safety parts,

- rubber and elastic parts,

- chassis parts,

- forgery and casting parts,

- electrical equipment and illumination systems,

- batteries,

- automotive glasses,

- seats.

www.journals.cz/
Automotive Industry (2004) analyzed and concluded that, although approximately 1,000 companies are involved in the automotive by products industry, number of the companies which apply acceptable manufacturing standards, produce original parts directly for the automotive manufacturing industry, compete with international markets through their export activities is 300 to 500 . Companies of the automotive by products industry are generally located in Istanbul, Bursa, and İzmir, and a review of these companies on the regional basis reveals the following percentages as to location of these companies: $75 \%$ in Marmara Region, $13 \%$ in Aegean Region, $7 \%$ in Inner Anatolian Region, and 5\% in other regions.

Turkish automotive by products industry, in which approximately 1000 companies are involved, has reached to a rather advanced level in terms of technology. Foreign Trade for Automotive Industry (1992-2003) concluded that majority of these companies hold internationally acceptable quality certificates (ISO 9000 , QS 9000 , ISO 14000 , ISO TS 16949 , and etc). The sectors carries out $70 \%$ of its exports to the member states of European Union.

According to the analysis of Automotive Industry: A Review of the Year 2002 (2003), automotive by products industry has an annual potential manufacturing capacity of $\$ 9$ Billions which can be realized if the automotive manufacturing sector operates with a capacity of $80 \%$, and domestic parts are utilized for $60 \%$ of the domestically produced vehicles. Therefore, automotive by products industry has a potential for an added value of $\$ 5.4$ Billions, export revenues of $\$ 3$ Billions, and investments of $\$ 450$ Millions per annum. Sector can provide direct employment for 100.000 individuals, and indirect employment for 500.000 individuals if the sector reaches to such a level. Although automotive by products industry has the necessary technological infrastructure, it can use only $30 \%$ of this potential at present.

\begin{tabular}{|c|c|}
\hline \multicolumn{2}{|l|}{ Contribution to the domestic economy (in Million US \$) } \\
\hline Manufacturing Value: & 9000 \\
\hline Added Value: & 3600 \\
\hline Investment Expenditures: & 450 \\
\hline Foreign currencies derived from export activities: & 3000 \\
\hline Tax Payments: & 2200 \\
\hline Direct Employment (Individual): & 150000 \\
\hline
\end{tabular}

Automotive by products industry has a close relationship with many sectors as follows:

- Agriculture,

- manufacturing industry (iron, steel, copper, aluminum, glass, paint, textile, and etc.),

- mining,

- tourism,

- transportation,

- construction, 
- defense,

- services (banking, insurance, and etc.).

Therefore, increase in production volume of automotive by products industry creates a significant demand for other sectors, and leads to an increase in production volume, employment level, and added values of these sectors.

Automotive Industry (2004) analyzed and concluded that, automotive by products industry has a high flexibility in terms of revenue and size, an an increase in the gross domestic product and population by $1 \%$ causes an increase in the production volume and added value of this sectors by over $1 \%$. Thanks to this character, automotive by products industry is very important for Turkey whose population and revenue level increase continuously. Because one of the most important problems of Turkey is to create employment opportunities for a fast-growing population. Turkey has to make investments, and grow larger due to this reason.

Export to the developed markets is one of the major strategic objectives of the automotive by products industry upon meeting the requirements for parts of export vehicles which are produced, or planned to be produced in Turkey, and obtaining of necessary quality assurance certificates. Companies of the automotive by products industry should attempt to improve quality of their products, management, and employees, and also improve cost and delivery processes.

It is required to ensure a full integration between the main industry and by products industry for the purpose of increasing competitive power and sustainability of the automotive industry.

According to the article A Research of Automotive Industry (2001), Şan concluded that "by products" used in manufacturing processes are developed in cooperation with the main industry and by products industry starting from design activities. This process is required for a reliabilitybased cooperation between the main industry and by products industry. By products industry may be able to make investment decisions for a long term cooperation in an environment of reliability. Technical integration allows both parties to join their powers, and use their resources in a more rational manner in terms of R\&D activities. It also leads to development of competitive products and technologies.

Technical integration supports specialization of the by products industry, and helps development of custom products. An environment of reliability supports establishment of capacities for production at economic scales for the by products industry. However, potential of the domestic by products industry could not be used sufficiently since main industry companies seek licenses, and foreign partners for many projects.

It appears as a prerequisite that sales prices of products intended for the main industry should be lower than those applicable in Europe.

Although degree of significance attached to the competitive priorities varies from one company to other, reliability of deliveries has become another prerequisite of the competition in the automotive sector where timely production and supply is valid.

Quality has become another competitive priority. Quality appears to be the second most important priority after delivery in the form of compliance to specifications, reliability, and durability.

Factors which allow a competitive production in the automotive by products industry are listed below in order of their importance:

www.journals.cz/
- Production at economic scales,

- cost of raw and auxiliary materials,

- labor costs,

- financing costs,

- quality,

- energy costs,

- shipment.

Following structural changes shall occur in the by products industry as a result of the technological changes in the automotive sector.

Technological innovations appear in vehicles as a result of ever increasing demands and requirements of customers as well as impacts of manufacturers thereon. However, legislation bodies, environmental lobbies, and social advancements shall also have a determinative factor on the speed of innovations.

Before all, pressure on the sector for competition and costs forces automotive manufacturers, first level by products companies, and manufacturing equipment suppliers to take necessary measures in the production and assembly processes for the purpose of decreasing costs. Additionally, external factors such as more strict laws and regulations governing environmental protection and exhaust wastes also play an important role in addition to minimizing use of raw materials. Furthermore, individual requirements of customers are a factor which triggers innovations. Functionality in comfort, prestige, safety, communication, individuality, and etc. leads to various innovations in manufacturing of vehicles.

Innovations shall take place through use of electronics technologies, and new material and new production technologies in the first place. Examples are electronic parts which focus on the fields of chassis, motor, and transmission systems, and cover all the modules of vehicles. This development represents a business volume of Eur 3.5 Billions only in Europe. For the purpose of decreasing complexity and costs, restrictions on modules and systems as well as relationships among them shall be defined again in the automotive sector. This situation shall cause a major change in the structure of the by products sector in the next decade. Newcomers of the market shall dominate the profitable segments of the market.

Demands of the automotive sector for both production process and final products lead to utilization of new materials, innovations in production technologies, and new concepts. Before all, light structural concepts and more economical production processes are demanded. Everincreasing utilization of aluminum and synthetic materials depends largely on light steel structures in the course of a development process. An ever-increasing competition shall take place in the fields of materials and production processes. Change which shall occur in the field of mechanical engineering shall lead to important structural changes in the by products industry.

In the automotive industry being dominated by e-commerce solutions heavier in the course of time, it is a fundamental requirement to have functional information technology architectures with network capabilities in order to survive. It is needed to anticipate a system which allows automation of the processes, and data communication between all the parties involved in the supply chain (from the by products industry to producers and end users), and consider future technologies. 
Weak aspects of the automotive by products industry

- High production costs due to low capacity utilization,

- excessive number of companies and excess capacity,

- inadequacy of financing instruments required to support the by products industry,

- failure to provide real and regular supports to the by products companies due to uncertainty and inadequacy of the definition of small and medium size enterprises,

- lack of competitive power due to insufficient training at some enterprises,

- loss of qualified labor and lack of motivation due to frequent economic crises,

- lack of competitive power suffered by some enterprises due to insufficiency of "Quality Management Systems" at the international level,

- lack of documentation concerning technical regulations, physical infrastructure, and organization,

- high energy input costs, and unreliable supply of utilities.

Aspects of the automotive by products industry which need improvement

- Lack of strategies for sectoral developments, and uncertain vision,

- lack of sufficient specialization and step manufacturing in Turkey when compared to the global developments, lack of system and module suppliers,

- failure of the by products industries to devote sufficient resources to R\&D activities and technological investments due to price policies applied by dependent main industry customers,

- failure to benefit from the potential of cooperation among companies in the fields such as communication, promotion, training and etc.,

- level of production below the economic scales due to import-focused and unstable development, and failure reflect increasing unit costs to the main industry companies,

- availability of a car manufacturing capacity in excess of demand, and failure to make investments required for global competition,

- failure to adapt to industrial design, patent, and international standards in a sufficient manner,

- increase in volume of vehicles imported, and imbalance of foreign trade in favor of import due to production which depends on imported parts.

\section{Conclusion}

Based on the findings above, the following strategies are recommended on each fundamental area.

Corporate skills should be improved:

- To improve skills in the fields of electronics, software, and mecatronics,

- to improve skills for marketing and market researches

- to improve skills for supply chain management and project management,

- to develop skills for understanding and managing end users,

- to make improvements in understanding the brand value.

Organizational structures of the companies should be improved:

- Value chain depth optimization / asset optimization,

- adaptation to business processes with e-commerce capabilities,

www.journals.czl
- establishment of an information platform for e-business,

- economic services which can be used by anyone,

- new models for career, and profit-sharing with employees,

- activities where a cooperation is established in expensive fields such as promotion and marketing.

New financing models should be applied:

- To establish external financing models through dialogues with international potential financing institutes,

- a new profit model (internal financing),

- to extend capabilities for mergers, takeovers, and investment disposals,

- to improve facilities offered by Eximbank.

Technologies and new skills should be integrated to the electronics and software fields:

- Each supplier should be able to identify any possible effects on its own module and the function within complete concept in a clear manner,

- review of the potential for changes in the module/system, and development of self skills in this field by means of electronics and software,

- creation of electronic skills,

- measures concerning personnel staff,

- alliances with partner organizations,

- establishment of a suitable organizational infrastructure, and transition to the functional organization to departmental project organization,

- introduction of open data and project management systems,

- performance of a value analysis for optimization of products and processes.

New market strategies should be identified:

- A clear orientation on achievement strategies and profit models

- getting prepared for future steps in terms of globalization,

- comparison with each other and with the best is a prerequisite of optimization,

- joint efforts for efficient promotion and sales in a costeffective manner,

- to conduct a SWOT analysis to identify the fields where it is required to provide a strategic training, and create an awareness for the purpose of enabling them to understand their present positions, and where it is required to improve business strategies.

Suppliers should be directed to one of the following five business fields in future in a clear manner:

- High Volume Suppliers - suppliers of standard parts, high volume,

- suppliers of New Markets - a small portion of the market. Separation via specialist technologies,

- part Specialists / Innovators - high market volume, separation via certain (technological) additional functions,

- module and System Specialists - development and production of complete systems including management of auxiliary suppliers,

- system Gatherers - development and integration of various modules and systems - development of joint strategies with original part manufacturers for new models and concepts.

Cooperation and initiatives up to integration of systems should be supported through establishment of system and module groups. 
To initiate and implement development of system and module partnerships among companies. Regular meetings are needed as in the case of standardization (for example, German Institute of Standards DIN). A central organization should manage this process through a portal.

To initiate the process of concentration concerning suppliers, and provided training to the suppliers in this respect. Active cooperation should be established on the international level.

To support a structural change in the by products industry through encouragement of automotive suppliers to enter into international cooperation. (for example, leverage of $R \& D$, procurement, manufacturing, and sales to an international platform, establishment and implementation of working groups in the fields of environment/recycling.)

Automotive by products industry should be encouraged to carry out quality manufacturing processes by means of environment-friendly technological applications, and special working groups with a focus on environmental protection and marketing should be established for this purpose.

Regulatory arrangements should be carried out in respect of recycling of harmful and chemical wastes, which are included in scope of environmental protection, during both manufacturing and utilization of products the industry of automotive and vehicle by products as well as other relevant aspects of the industry.

Present positions of companies should be improved as per the global technological developments:

- To carry out a comprehensive promotion of the capabilities of Turkish automotive by products industry by means of marketing campaigns,

- to improve capabilities of suppliers,

- to encourage international cooperation through both local and international dialogues,

- to strengthen system capabilities of suppliers through cooperation,

- to gather know-how within businesses and the country through partnerships for future strategic products,

- to use existing financing programs for the purpose of introducing Turkey as a country of automotive by products industry, and make salary structure, business and working hours flexible,

- to eliminate bureaucratic obstacles,

- to support the companies through supply of capital,

- to encourage structural changes instead of attempting to repair existing structures,

- to initiate partnerships and joint investments instead of supporting the cooperation among local medium size companies, or waiting for acquisition thereof by national and international large size companies,

- to manage the cooperation among local automotive manufacturers instead of acceptance by the suppliers of the by products industry of indefinite adaptation processes as a result of pressures exerted by the automotive industry and the competition,

- to apply promotional measures in a target-oriented manner based on requirements of future structures instead of creating facilities on the basis of existing structure,

- to focus money and campaigns on the key points in a target-oriented manner instead of extending budgets,

- to appoint existing employees of the relevant authorities to the key points instead of increasing number of employees,

www.journals.cz/
- to ensure that potential partners (for example, internal official entities such as trade associations, trade foundations in addition to VDA and regional business environments) take part in initiatives in a timely and cooperative manner for the purpose of preventing waste of individual initiatives.

\section{REFERENCES}

1. Monthly Statistical Data Bulletin. Ankara, 2004. www.osd.org.tr. 2. Automotive Industry: A Review of the Year 2002. Ankara, 2003. www.osd.org.tr.

3. Automotive Industry. Ankara, 2004. www.osd.org.tr.

4. Foreign Trade for Automotive Industry. Ankara, 1992-2003.

5. Şan, İlhami. A Research of Automotive Industry. Ankara: Turkish Ministry of Industry and Commerce, 2001. 